\title{
Nonequilibrium transport response from equilibrium transport theory
}

\author{
V. M. García-Suárez* and J. Ferrer \\ Departamento de Física, Universidad de Oviedo, E-33007 Oviedo, Spain, Nanomaterials and Nanotechnology Research Center \\ (CINN, CSIC - Universidad de Oviedo), E-33007 Oviedo, Spain, and Department of Physics, Lancaster University, \\ Lancaster LA1 4YB, United Kingdom
}

(Received 27 July 2012; revised manuscript received 14 September 2012; published 27 September 2012)

\begin{abstract}
We propose a simple scheme that describes accurately essential nonequilibrium effects in nanoscale electronics devices using equilibrium transport theory. The scheme, which is based on the alignment and dealignment of the junction molecular orbitals with the shifted Fermi levels of the electrodes, simplifies drastically the calculation of current-voltage characteristics compared to typical nonequilibrium algorithms. We probe that the scheme captures a number of nontrivial transport phenomena such as the negative differential resistance and rectification effects. It applies to those atomic-scale junctions whose relevant states for transport are spatially placed on the contact atoms or near the electrodes.
\end{abstract}

DOI: 10.1103/PhysRevB.86.125446

PACS number(s): 73.63.-b, 05.70.Ln, 72.10.-d

Nanoscale devices tend to suffer from a serious lack of reproducibility from one research group to another, and from a lack of durability and robustness. ${ }^{1,2}$ However, the consistent and thorough work of a range of experimental groups has allowed researchers to reach some consensus on the conductance values and variability of a few specific junctions. ${ }^{3-5}$ In addition, the development of simulation codes based on a combination of density functional theory (DFT) and the nonequilibrium Green's function formalism (NEGF) ${ }^{5-12}$ has enabled theoreticians to assist the above experiments with theoretical insights and predictions. However, the size and complexity of the experimentally active part of the junction is typically too large, and the above codes need to assume a number of simplifications regarding the size, geometry, number of feasible atomic arrangements, and the electronic correlations. Recently, some of the above codes have been combined with classical force field programs, enabling the simulation of much larger systems and the sampling of a much wider set of atomic arrangements. ${ }^{13,14}$ Nevertheless, present current-voltage $I-V$ characteristics are still computed using NEGF techniques, which are rather cumbersome and extremely computer hungry. In other words, the NEGF is a serious bottleneck hindering the deployment of realistic transport simulations at the nanoscale. In contrast, equilibrium transport techniques are much simpler, better founded on physical grounds, and computationally drastically less demanding. ${ }^{6}$ However, they are completely unable to reproduce current-voltage curves of nanoscale devices. ${ }^{15}$ This is so because these techniques assume that the electronic states and the Hamiltonian at the junction do not change under the application of a voltage. However, a voltage bias drives a flow of electrons into and out of the junction, driving the device out of equilibrium and possibly even changing its quantum state and sometimes even its atomic arrangement. For example, the electric field originated by the voltage bias can also shift the energy position of the molecular levels by the Stark effect, depending on the polarizability of each molecular level or on whether the molecule has itself a polar nature. ${ }^{16,17}$

We show here a suitable modification of equilibrium transport techniques that accounts for a large set of nonequilibrium transport phenomena such as negative differential resistance $(\mathrm{NDR})^{18}$ or rectification effects. The performance of our method depends on the energy position, electrode coupling, and spatial localization of the molecular orbitals that are responsible for the transport properties. This works especially well for systems where the closest states to the Fermi level (HOMO or LUMO) are located at the contact atoms or near the electrodes. We discuss below the physical mechanisms at work for several molecular-scale functions. Our scheme enables the simulation of $I-V$ curves which compare favorably to those obtained using NEGF techniques. As a consequence, we also propose that a number of transport phenomena regarded up to now as genuine manifestations of nonequilibrium behavior of a junction could be recognized as quantitative but not qualitative modifications of its equilibrium state.

We use Caroli's scheme ${ }^{20}$ and split the device into left and right electrodes, and an extended molecule. The extended molecule includes several principal layers of the electrodes, as well as the molecule itself. The full Hamiltonian $\mathcal{H}$ can be written as

$$
\left(\begin{array}{ccc}
\mathcal{H}_{\mathrm{L}}+\frac{e V}{2} \mathcal{S}_{\mathrm{L}} & \mathcal{H}_{\mathrm{LM}}+\frac{e V}{2} \mathcal{S}_{\mathrm{LM}} & 0 \\
\mathcal{H}_{\mathrm{ML}}+\frac{e V}{2} \mathcal{S}_{\mathrm{ML}} & \mathcal{H}_{\mathrm{M}}[\rho] & \mathcal{H}_{\mathrm{MR}}-\frac{e V}{2} \mathcal{S}_{\mathrm{MR}} \\
0 & \mathcal{H}_{\mathrm{RM}}-\frac{e V}{2} \mathcal{S}_{\mathrm{RM}} & \mathcal{H}_{\mathrm{R}}-\frac{e V}{2} \mathcal{S}_{\mathrm{R}}
\end{array}\right)
$$

where the matrices $\mathcal{H}_{\mathrm{L}}, \mathcal{H}_{\mathrm{R}}, \mathcal{H}_{\mathrm{LM}}, \mathcal{H}_{\mathrm{RM}}$, and their corresponding overlap matrix blocks, indicate respectively the leftand right-hand side leads Hamiltonians and the coupling matrices between the leads and the extended molecule. The matrix elements of the extended molecule Hamiltonian $\mathcal{H}_{\mathrm{M}}^{i, j}$ run over all the orbitals residing in the extended molecule region. They depend on the nonequilibrium density matrix $\rho$, which must be calculated self-consistently for each voltage $V$ using cumbersome and computationally very demanding NEGF techniques. ${ }^{5,7-12}$

We propose here that in many situations $\rho$ and its derived $\mathcal{H}_{\mathrm{M}}[\rho]$ need only be computed at zero voltage, and that the effect of a finite bias can be accounted for by the alignment or dealignment of the molecular orbitals at the extended molecule region with the energy levels of the electrodes (which are shifted by $\pm e V / 2$ by the bias voltage). Mathematically, we apply a simple shift to the Hamiltonian matrix elements from $\mathcal{H}_{\mathrm{M}}$ to $\mathcal{H}_{\mathrm{M}}+e V_{i} S_{\mathrm{M}}$, where the local shifts $V_{i}$ depend on the junction nature. For example, in a highly transparent junction, 
the shifts can be modeled by a linear voltage ramp connecting the matrix elements of the orbitals at the outermost layers. In contrast, most nanoscale junctions contain a molecule that is connected to the left and right electrodes by weaker links, which means the details of the coupling of the electrodes to the molecular orbitals responsible for the transport must be taken into account. Our scheme is specially suited for junctions where the HOMO and LUMO orbitals are spatially localized at the linker atoms joining the molecule with the electrodes. In this case, we take $V_{i}= \pm e V / 2$ for all orbitals starting at the outermost layers in the extended molecule and up to the linker atoms, and $V_{i}=0$ for all the remaining inner orbitals. We show now how these shifts allow one to mimic accurately a number of simple and not so simple junctions displaying nontrivial negative differential resistance, as well as rectification effects for some asymmetric molecules.

Notice that in NEGF simulations the left and right electrodes are defined as semi-infinite electron reservoirs so that a steady electron flow is established. The chemical potentials at the left and right electrodes are shifted by $\pm e V / 2$ and a permanent potential drop is produced somewhere in the extended molecule. These features are reproduced by our scheme. We have also checked that they are not reproduced by applying an electric field along the transport direction in equilibrium simulations of finite-sized systems that represent the extended molecule. This is so because in these later cases the junction has a finite length and equilibrium conditions are reestablished at the end of the simulation. We have verified that the applied electric field shifts initially the electronic states as in our scheme, but because the whole device has a common Fermi energy, a positive charge surplus accumulates at the negative electrode and vice versa, thereby screening the potential created by the electric field and moving back the energy levels towards their original positions.

We discuss first two simple examples to illustrate the scheme. These systems are made of two semi-infinite carbon chains terminated each by either a sulfur or a nitrogen atom and separated initially by a vacuum region of $3.7 \AA$. We apply a shift of $\pm e V / 2$ to each Hamiltonian matrix element at the extended molecule region up to and including the terminating atoms as proposed above. The left panels in Fig. 1 show the $I-V$ characteristics of the two junctions, which display a conventional ohmic behavior. However, these junctions show NDR when the terminating atoms are slightly displaced away from the chains. ${ }^{21}$ The $I-V$ curves of the two junctions are plotted in the right panels of Fig. 1. The NDR response shown is originated by a nontrivial energy shift and splitting of the resonances appearing in the transmission coefficients $T(E)$ as the voltage bias is ramped up. This nontrivial evolution of the transmission resonances is shown in the left panels of Fig. 2 and could be thought of as a genuine nonequilibrium effect at first sight. That this is so could also be inferred from the $I-V$ curves computed from the equilibrium equation,

$$
I=\frac{e}{h} \int_{-e V / 2}^{e V / 2} d E T(E),
$$

where $T(E)$ is calculated at zero voltage. Such $I-V$ curves are indeed quite different from those computed using standard NEGF techniques, as shown in the right panels of Fig. 1.

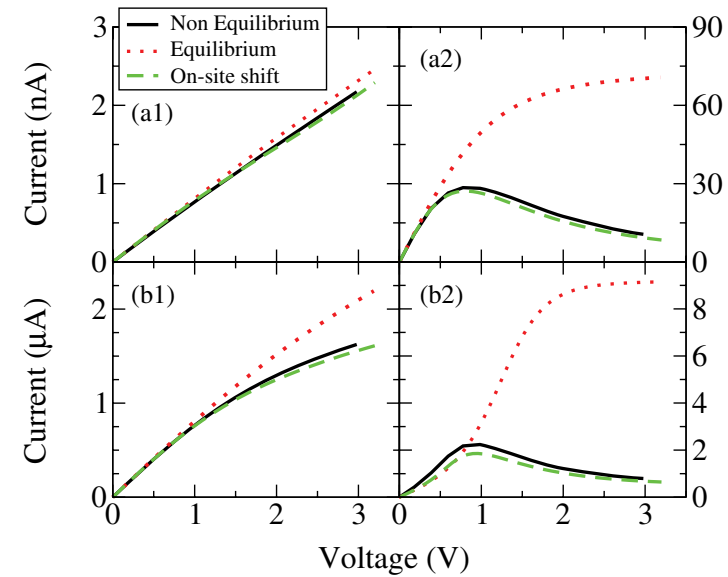

FIG. 1. (Color online) $I-V$ curves of a junction made of two carbon chains, each terminated by a nitrogen (a) or a sulfur atom (b). These two atoms are separated by a physical gap of $3.7 \AA$. Panels (a1) and (b1) correspond to fully relaxed coordinates. Panels (a2) and (b2) correspond to chains where the terminating nitrogen and sulfur atoms have been separated by $0.5 \AA$ away from their relaxed positions. Solid and dotted lines correspond to NEGF and equilibrium simulations, respectively. Dashed lines correspond to simulations using the scheme proposed in this article.

However, the figure also shows that Eq. (2) can produce extremely accurate results if $T(E)$ is calculated using a modified Hamiltonian $\mathcal{H}_{\mathrm{M}}$ that follows our scheme. Furthermore, the right panels in Fig. 2 show that the nontrivial bias evolution of the transmission resonances is also followed by our scheme with high fidelity. As a consequence, we propose that the resonance splitting and shift shown in the figure is only apparent. What actually happens is that the single resonance is cut off by an opposite shift of the on-site energy levels at opposite sides of the junctions, and is therefore not a genuine nonequilibrium effect.

The scheme proposed cannot be applied to all physical situations. An example where the scheme fails occurs when the molecular orbital responsible for transport (HOMO or

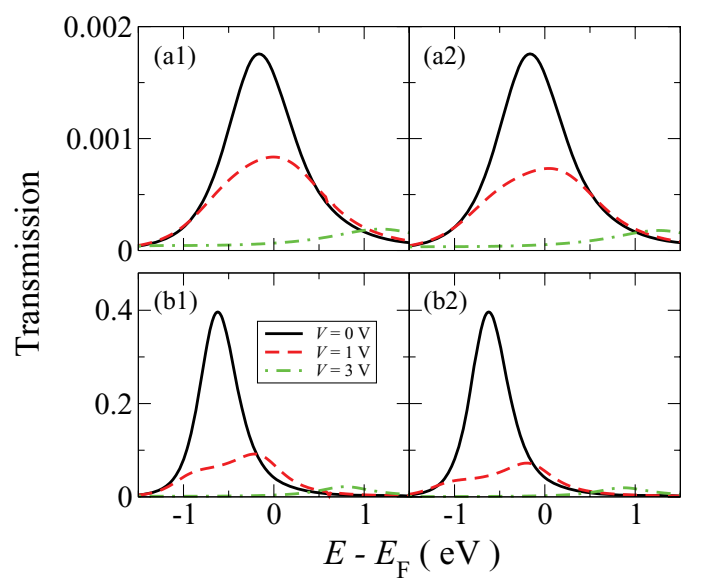

FIG. 2. (Color online) Transmission curves at different voltages for the carbon junctions shown in panels (a2) and (b2) of Fig. 1. Panels (a1) and (b1) correspond to a full NEGF calculation. Panels (a2) and (b2) correspond to the scheme proposed in this article. 
LUMO) is well localized in the central region of the molecule. In this case, the resonance associated with that molecular orbital could be pinned to the Fermi level of either the left or the right electrode. ${ }^{22}$ However, even in this scenario the scheme proposed improves slightly the $I-V$ curves compared to raw equilibrium simulations, because it takes into account a nonequilibrium mechanism that is present in all nanoscale junctions. We discuss next a series of molecular junctions where different polyyne derivatives are contacted by (001) gold electrodes to illustrate this argument. Results for the synthesis of the molecules and the transport properties of the devices have been reported in Ref. 23.

Figure 3(a) shows the $I-V$ curves of a pyridine-polyynepyridine junction, where the polyyne has $n=3$ carbon pairs. Following our scheme, we apply a rigid shift to the orbitals of the extended molecule up to and including the nitrogen atoms at the two pyridine rings. The resulting $I-V$ curve, plotted in Fig. 3(a), shows that the scheme fails calamitously to describe the NDR response of this junction, even though it improves the equilibrium-based calculation of the $I-V$ curve, which always increases. Furthermore, we have tested that extending the shift up to the inner carbon atoms in the pyridine rings does not improve matters. In contrast, the scheme provides reasonably accurate curves for similar polyyne-based junctions where the pyridines are replaced by sulfur atoms [Fig. 3(b)] or by

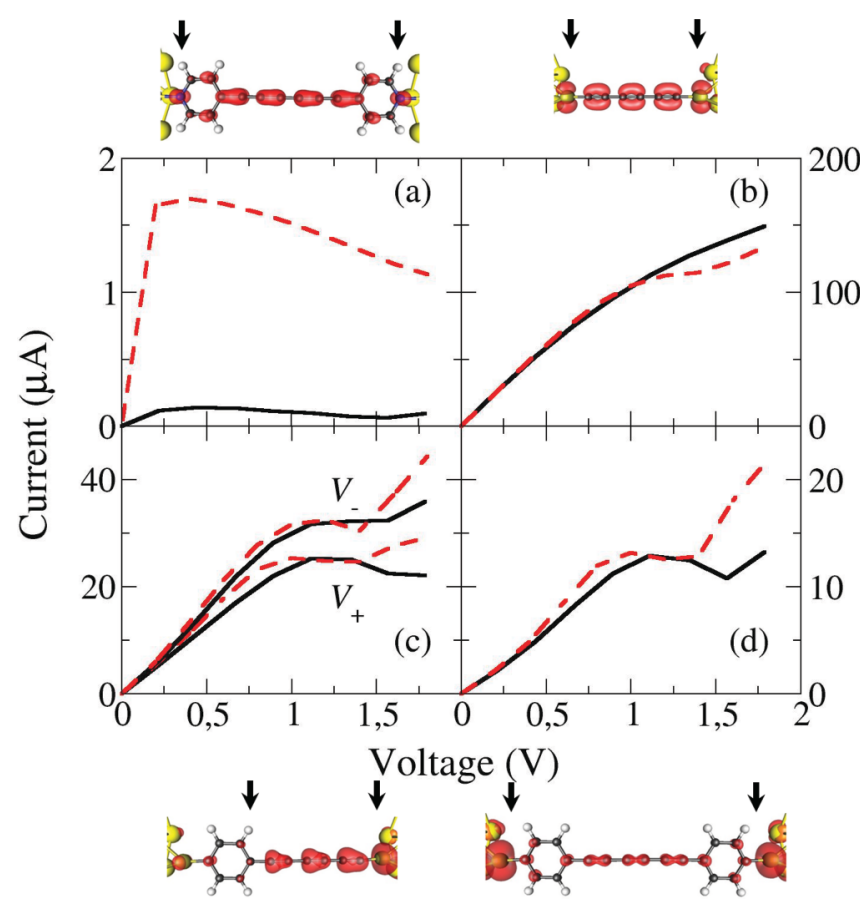

FIG. 3. (Color online) $I-V$ curves of gold (001) junctions sandwiched by pyridine-polyyne-pyridine (a), sulfur-polyyne-sulfur (b), sulfur-benzene-polyyne-sulfur (c), and sulfur-benzene-polyynebenzene-sulfur (d) molecular wires, where the polyyne has $n=3$ carbon pairs. The molecules are sketched above or below each corresponding graphic, together with density contours that highlight the localization of the relevant molecular orbital. Solid lines correspond to nonequilibrium calculations, while dashed lines correspond to calculations performed with the scheme proposed in this article. The on-site energy levels have been shifted up to the atoms indicated by the arrows.

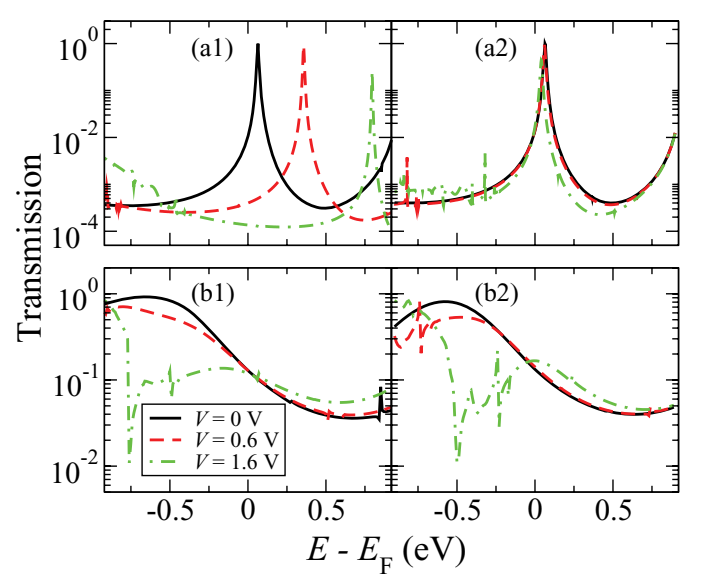

FIG. 4. (Color online) Transmission curves at different voltages for the pyridine-polyyne-pyridine (top panels) and sulfur-benzenepolyyne-benzene-sulfur (bottom panels) junctions shown in panels (a) and (c) of Fig. 3. Panels (a1) and (b1) correspond to a full nonequilibrium calculation. Panels (a2) and (b2) correspond to the scheme proposed in this article.

sulfur-benzene rings [Figs. 3(c) and 3(d)]. The corresponding $T(E)$ curves for the pyridine-polyyne-pyridine and sulfurbenzene-polyyne-benzene-sulfur junctions, calculated with NEGF and with our scheme, are shown in Fig. 4. This figure shows how in the first case, a sharp resonance exists, which moves to higher energies as the bias increases while maintaining its shape. In contrast, our scheme predicts that the resonance does not shift with voltage because it is placed at the center of the molecule, as we show in Fig. 4(a2). The impact of a finite bias in the second junction, however, is manifested in the destruction of the HOMO resonance, shown in Fig. 4(b), which is now well captured by our scheme.

The criterion to discern when the proposed scheme can be used is related to the nature and features of the molecular level responsible for the transport properties. Those are its spatial placement in the molecule, its energy positioning as well as its coupling to the electrodes. For the pyridine-polyyne-pyridine molecule, the LUMO orbital is made essentially of carbon $\pi$ states and is located on the central part of the molecule. When the bias is applied, the voltage drop does not occur at this orbital because the highly conjugated character of the molecule screens the potential. Therefore the applied bias has a small impact in the LUMO orbital and as a result the shape of the associated transmission resonance does not change. The resonance shifts up in energy because there is a net transfer of charge from the electrodes towards the molecule. This moves all levels upwards and pins the LUMO orbital to the negative electrode. For the sulfur-benzene-polyynebenzene-sulfur junction, the relevant orbital is the HOMO, which is mostly located at the sulfur atoms. The atomic sulfur states couple across the molecule and produce bonding and antibonding states. When the bias is ramped up, those sulfur states follow the chemical potential of each electrode because they are strongly coupled to the leads. As a consequence, the bonding and antibonding molecular levels are destroyed. This is seen in the transmission as a reduction of the HOMO-related resonance, which produces an NDR response. We note that this NDR behavior does not occur if the molecule does not 
contain the benzene rings as shown in Fig. 3(b). In this case the coupling between the sulfur states and the carbon chain is much stronger. This increases the hybridization between them, keeps the integrity of the bonding and antibonding states, and decreases the impact of the shifts. An intermediate case between these two junctions corresponds to the asymmetric sulfur-benzene-polyyne-sulfur molecule shown in Fig. 3(c). This junction gives different $I-V$ characteristics for each bias polarity, producing a rectifying response. In this case the relevant orbital is again the HOMO state, which is mostly localized in the right sulfur atom and the carbon chain. The chain states are left unaffected when we ramp up the bias and apply the scheme to the sulfur on the right side and the benzene carbon atom closer to the chain. The scheme reproduces quite well both the NDR and the rectification effects.

The scheme proposed is specifically suited for junctions where the states are well localized at the linking atoms or near the electrodes, because then the frontier orbitals are strongly affected by the applied bias. Large junctions are difficult to simulate using NEGF techniques because they require huge amounts of computer resources and have frequently convergence problems. Our scheme simplifies the calculation of $I-V$ curves drastically, because it relies on equilibrium techniques, which are much simpler and computationally much less demanding. We have indeed verified that the $I-V$ curves corresponding to a series of graphene junctions showing
NDR response ${ }^{21}$ are accurately described with our scheme. The recipe proposed in this article can be used whenever the ground state at the molecule is not changed by the nonequilibrium conditions. In the electrostatic spin effect, for example, the spin multiplicity of the molecule is changed by the application of a finite bias, ${ }^{17}$ and as a result our scheme cannot be applied.

In summary, we propose in this article that current-voltage characteristics of a wide array of electronic junctions can be accurately described by a scheme that combines conventional equilibrium transport techniques with suitable shifts of energy levels up to the weak links existing in those junctions. This scheme can have drastic practical effects because equilibrium codes are much simpler and computationally more efficient than nonequilibrium codes. We have corroborated very large computational speed-ups in the above examples. As a consequence, our recipe opens a route towards realistic transport simulations comprising much bigger or complex systems than those that could be feasibly simulated with current NEGF techniques.

The research presented here was funded by the Spanish MICINN through Grant No. FIS2009-07081 and by the Marie Curie network nano CTM. V.M.G.S. thanks the Spanish Ministerio de Economía y Competitividad for a Ramón y Cajal fellowship (Grant No. RYC-2010-06053). *vm.garcia@cinn.es

${ }^{1}$ Assessment of the Potential and Maturity of Selected Emerging Research Memory Technologies. Workshop and ERD/ERM Working Group Meeting, International Technology Roadmap for Semiconductors (April 6-7, 2010).

${ }^{2}$ J. R. Heath, Annu. Rev. Mater. Res. 39, 1 (2009).

${ }^{3}$ R. L. McCreery and A. J. Bergren, Adv. Mater. 21, 4303 (2009).

${ }^{4}$ K. Moth-Poulsen and T. Bjornholm, Nature Nanotech. 4, 551 (2009).

${ }^{5}$ J. C. Cuevas and E. Scheer, Molecular Electronics: An Introduction to Theory and Experiment (World Scientific, Singapore, 2010).

${ }^{6}$ S. Datta, Electronic Transport in Mesoscopic Systems (Cambridge University Press, Cambridge, 2007).

${ }^{7}$ J. Taylor, H. Guo, and J. Wang, Phys. Rev. B 63, 245407 (2001).

${ }^{8}$ Y. Xue, S. Datta, and M. A. Ratner, Chem. Phys. 281, 151 (2002).

${ }^{9}$ M. Brandbyge, J.-L. Mozos, P. Ordejón, J. Taylor, and K. Stokbro, Phys. Rev. B 65, 165401 (2002).

${ }^{10}$ J. J. Palacios, A. J. Pérez-Jiménez, E. Louis, E. San Fabián, and J. A. Vergés, Phys. Rev. B 66, 035322 (2002).

${ }^{11}$ A. Pecchia and A. Di Carlo, Rep. Prog. Phys. 67, 1497 (2004).

${ }^{12}$ A. R. Rocha, V. M. García-Suárez, S. Bailey, C. Lambert, J. Ferrer, and S. Sanvito, Phys. Rev. B 73, 085414 (2006).
${ }^{13}$ C. F. Sanz-Navarro, R. Grima, A. García, E. A. Bea, A. Soba, J. M. Cela, and P. Ordejón, Theor. Chem. Acc. 128, 825 (2011).

${ }^{14}$ P. Makk, D. Visontai, L. Oroszlańy, D. Zs. Manrique, Sz. Csonka, J. Cserti, C. J. Lambert, and A. Halbritter, Phys. Rev. Lett. 107, 276801 (2011).

${ }^{15}$ C. Y. Yam, X. Zheng, G. H. Chen, Y. Wang, T. Frauenheim, and T. A. Niehaus, Phys. Rev. B 83, 245448 (2011).

${ }^{16}$ M. Diefenbach and K. S. Tim, Angew. Chem. 46, 7640 (2007).

${ }^{17}$ N. Baadji, M. Piacenza, T. Tugsuz, F. Della Sala, G. Maruccio, and S. Sanvito, Nat. Mater. 8, 813 (2009).

${ }^{18} \mathrm{NDR}$ from equilibrium transport properties has already been observed in model molecular wires using a mean-field Hubbard model. ${ }^{19}$ The NDR was produced by voltage-induced localization.

${ }^{19}$ V. Mujica, M. Kemp, A. Roitberg, and M. Ratner, J. Chem. Phys. 104, 7296 (1996).

${ }^{20}$ C. Caroli, R. Combescot, P. Nozieres, and D. Saint-James, J. Phys. C 5, 21 (1972).

${ }^{21}$ D. J. Carrascal, V. M. García-Suárez, and J. Ferrer, Phys. Rev. B 85, 195434 (2012)

${ }^{22}$ V. M. García-Suárez and C. J. Lambert, Nanotechnology 19, 455203 (2008).

${ }^{23}$ C. Wang, A. S. Batsanov, M. R. Bryce, S. Martín, R. J. Nichols, S. J. Higgins, V. M. García-Suárez, and C. J. Lambert, J. Am. Chem. Soc. 131, 15647 (2009). 